\title{
Simultaneous in situ characterisation of bubble dynamics and a spatially resolved concentration profile: a combined Mach-Zehnder holography and confocal Raman-spectroscopy sensor system
}

\author{
Jajnabalkya Guhathakurta ${ }^{1}$, Daniela Schurr ${ }^{2}$, Günter Rinke ${ }^{2}$, Roland Dittmeyer ${ }^{2}$, and Sven Simon ${ }^{1}$ \\ ${ }^{1}$ Institute for Parallel and Distributed Systems (IPVS), Department of Parallel Systems, Universitätsstr. 38, \\ 70569 Stuttgart, Germany \\ ${ }^{2}$ Institute for Micro Process Engineering (IMVT), Karlsruhe Institute of Technology, \\ Hermann-von-Helmholtz-Platz 1, 76344 Eggenstein-Leopoldshafen, Germany
}

Correspondence to: Jajnabalkya Guhathakurta (jajnabalkya.guhathakurta@ipvs.uni-stuttgart.de)

Received: 10 October 2016 - Revised: 5 February 2017 - Accepted: 16 March 2017 - Published: 23 May 2017

\begin{abstract}
For a reaction between a gaseous phase and a liquid phase, the interaction between the hydrodynamic conditions, mass transport and reaction kinetics plays a crucial role with respect to the conversion and selectivity of the process. Within this work, a sensor system was developed to simultaneously characterise the bubble dynamics and the localised concentration measurement around the bubbles. The sensor system is a combination of a digital Mach-Zehnder holography subsystem to measure bubble dynamics and a confocal Raman-spectroscopy subsystem to measure localised concentration. The combined system was used to investigate the chemical absorption of $\mathrm{CO}_{2}$ bubbles in caustic soda in microchannels. The proposed set-up is explained and characterised in detail and the experimental results are presented, illustrating the capability of the sensor system to simultaneously measure the localised concentration of the carbonate ion with a good limit of detection and the 3-D position of the bubble with respect to the spot where the concentration was measured.
\end{abstract}

\section{Introduction}

The main application of gas dispersion in industry is to transfer gas into liquid, for instance in order to run a chemical reaction (Deen et al., 2012; Jakobsen, 2008). In the chemical industry, bubble columns are a widely used tool, especially for reactions that require a large liquid hold-up and long liquid-phase residence times without the need for a narrow residence time distribution (Deckwer, 1992; Deen et al., 2012; Jakobsen, 2008). However, a realistic description and prediction of industrially relevant reactive bubbly flows has been a challenge until now (Deckwer, 1992; Deen et al., 2012; Jakobsen, 2008). Thus, the complex problem is scaled down to single bubbles and bubble chains in order to gain insight into the processes of hydrodynamics and mass transfer in reactive two-phase flows and how they influence the conversion and selectivity of the desired products. In this paper, a Taylor flow set-up is studied. The Taylor flow is a gasliquid flow in microchannels that consists of elongated bubbles that take up almost the whole cross section of the channel separated by liquid slugs (Sobieszuk et al., 2012; Kashid et al., 2011; Gupta et al., 2010; Angeli and Gavriilidis, 2008). To enable a close look into the difficult interactions of multiphase flows, a noninvasive measurement technique is required to avoid the disruption of the hydrodynamic flow field. Additionally, there is a need for two different measurement techniques. On the one hand, the fluid dynamics should be monitored to gain information about the bubble size and form and to obtain information about the velocity trajectories of the gaseous and the liquid film. On the other hand, a sensitive tool for quantitative measurements of the concentration is necessary. Both should be operable with high temporal and spatial resolution to monitor highly anisotropic and transient conditions. 
For the first aspect, the bubble dynamics, real-time shadowgraphy has emerged as the most common tool with advances in high-speed cameras. Thoroddsen et al. (2008) and Versluis (2013) present overviews of the various techniques of shadowgraphy and its application in the domain of fluid dynamics. It involves taking shadow images of objects illuminated by light sources, such as high-power LEDs (Gebauer et al., 2016) or lasers with different beam geometries (Lindken and Merzkirch, 2002; Sathe et al., 2010; Tokuhiro et al., 1999), at very high frame rates. Although this technique is simple and robust, it suffers from a few limitations. The foremost limitation of shadowgraphy is the need for the object to be in focus. The bubbles move frequently in and out of the focal plane, and this limits their detection as they appear defocussed in the image and its surface position cannot be easily extracted. Secondly, if there are multiple objects present with overlapping positions along the direction of light, they appear as single objects in the shadow image and cannot be distinguished from each other. As this is often the case with bubbles, shadowgraphy might result in measuring the wrong geometric dimensions and the number of the objects in reality. Lastly, with this technique only the 2-D position of the object can be determined in the focal plane. In order to determine the 3-D position of the object, shadowgraphy must be performed from at least two different optical axes (preferably perpendicular to each other). In many cases, it is physically not possible to have multiple optical axes due to spatial constraints and limited optical access.

The use of digital holography techniques (Kim, 2010; Orlov et al., 2010; Desse and Olchewsky, 2016), like MachZehnder off-axis holography (Toker, 2016), eliminates the disadvantages of the shadowgraphy technique at the expense of more computation power for reconstructing the holograms. For this technique, lasers are used as coherent light sources to record the phase and intensity of the light transmitted through the object as interference patterns in the holograms. This is achieved by splitting the coherent beam into two parts, and only one part is allowed to pass through the object before finally recombining them to capture the hologram. Afterwards, the holograms are reconstructed and digitally focussed to obtain a sharp image and the 3-D position of the object. Lebrun et al. (2011) and Tanaka and Murata (2012) have used similar holographic techniques to measure the size and position of bubbles. However, both works present an in-line holographic technique which suffers from distortion caused by overlapping of the twin image (Mico et al., 2010). On the other hand, the digital Mach-Zehnder off-axis holography presented in this paper allows for the digital separation of the twin image and is capable of extracting the sharp edges of the bubbles.

For the second aspect, the concentration measurement, only noninvasive techniques can be used as any invasive method alters the hydrodynamics within the system. Confocal Raman spectroscopy provides high spatial resolution along with high selectivity for concentration measurement.
It is a measurement technique commonly used in chemistry, since it provides information concerning the chemical bonds and the symmetry of the molecules. Within the last decades, the method has been further developed for in situ process analysis and reaction monitoring in research, pharmaceutical operations and the chemical industry (Fletcher et al., 2003; Kessler et al., 2016; Lewis, 2001; Vankeirsbilck et al., 2015; Rantanen, 2007). At the Institute for Micro Process Engineering, previous work was done on the development of an in situ laser Raman system to examine mixing processes (Rinke et al., 2011) and the kinetics of the oxidation of cyclohexane (Fräulin et al., 2013, 2014). For gas-liquid flows, the Raman set-up had to be enhanced by replacing the previously used continuous-wave laser by a pulsed laser as an excitation source.

In this paper, we present a sensor system to simultaneously measure the bubble dynamics and the spatially resolved concentration. The bubble position in the sensor system was determined using digital Mach-Zehnder holography, while the concentration was measured using confocal Raman spectroscopy. Both of the techniques were synchronised and operated simultaneously. An analysis system combining laser Raman spectroscopy and holography was previously used by Ferrara et al. $(2015,2016)$ as well as by Pavillon and Smith (2015) to study biological samples, e.g. the morphology and molecular composition of cells and cell components. In order to avoid the disruption of the immobilised organic sample, they used continuous-wave Nd:YAG lasers with low power. In contrast to this, we use high-power pulsed lasers to provide the short integration times that are necessary for transient two-phase flows.

\section{A combined Mach-Zehnder holography and confocal Raman-spectroscopy sensor system set-up}

The sensor system consists of two subsystems, as depicted on the left side of Fig. 1, one for the bubble dynamics measurement and another for localised concentration measurements below the bubble. The Taylor flow set-up investigated in this paper is illustrated on the right side of Fig. 1. It includes a transparent microchannel $(O)$ made up of borosilicate glass with a nominal rectangular cross section $O^{\prime}$ (shown in the top left of Fig. 1) of $2 \times 2 \mathrm{~mm}$ and a wall thickness of $0.3 \mathrm{~mm}$. The contacting of the gas and the liquid phase occurs directly at the bottom of the rectangular microchannel with a small capillary which serves as a needle injector. It provides a vertical upward flow of alternating bubbles and slugs. The liquid is metered by a syringe pump. A mass flow controller is used to adjust the gas flow rate. The first tests were conducted using a non-reactive Taylor flow between air and water with varying flow rates. For the chemical absorption of $\mathrm{CO}_{2}$ in caustic soda, the flow rates were set to $0.5 \mathrm{~mL} \mathrm{~min}^{-1}$ for the liquid phase and $17 \mathrm{~mL} \mathrm{~min}^{-1}$ for the gaseous phase. 


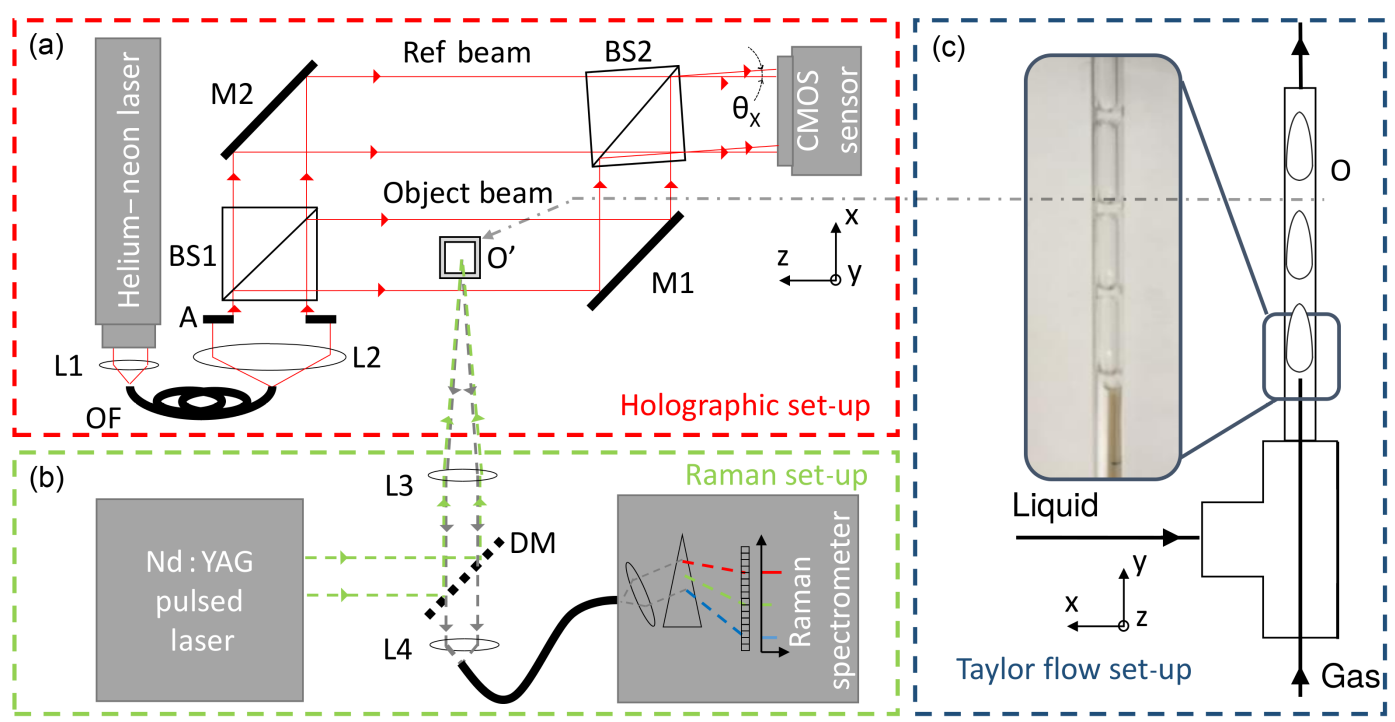

Figure 1. A combined Mach-Zehnder holography and confocal Raman-spectroscopy sensor system set-up. (a) The digital Mach-Zehnder holography subsystem, (b) the confocal Raman-spectroscopy subsystem (c) and the Taylor flow set-up. $O$ is the side view of the microchannel and $O^{\prime}$ is the top view viz. the cross section of the capillary.

The sensor system is composed of the digital MachZehnder holography subsystem for the measurement of bubble dynamics, as illustrated in Fig. 1 (top left), and the confocal Raman spectroscopy for the localised concentration measurement, also shown in Fig. 1 (bottom left). The Cartesian coordinate system in the set-up is also introduced in Fig. 1. These two optical subsystems are aligned to the two perpendicular optical axes of the rectangular microchannel, i.e. the optical axis along the $z$ direction of $O^{\prime}$ is used by holography and the optical axis along the $x$ direction of $O^{\prime}$ is used by Raman spectroscopy. This arrangement facilitates the simultaneous measurement of the position of a rising bubble and the concentration at a fixed calibrated point within the microchannel. The distance between the spot where the concentration measurement is performed and the lowermost point of the bubbles is extracted from the reconstructed hologram. A set of measured data points with the concentration measurement and the correlated distance to the bubble will provide the concentration profile of specific regions around the bubble.

\subsection{Digital Mach-Zehnder off-axis holography subsystem}

In this holographic technique, a laser beam is divided into two equal parts, but only one is made to pass through the object. Afterwards, both of the beams are directed and recombined to capture the interference pattern with a digital image sensor. This interference image, known as the hologram, encodes the intensity as well as the phase information about the object as intensity profiles. A simplified diagram of this holographic technique is shown in Fig. 1 (top left).
A helium-neon $(\mathrm{HeNe})$ laser $(\lambda=633 \mathrm{~nm})$ is chosen as the coherent light source due to its stable performance and high coherence length. The parallel beam of this laser is focussed into a monomode glass fibre, $\mathrm{OF}$, with a $4 \mu \mathrm{m}$ core diameter using the coupler L1. On the other end of the fibre, the diverging beam is collimated to a beam with a diameter of $10.8 \mathrm{~mm}\left(1 / e^{2}\right.$ level of the Gaussian intensity distribution) using the fibre collimator L2. The ends of this fibre have a cut-off angle of $8^{\circ}$ to prevent reflection back into the laser. The small core diameter also acts as a pinhole within a spatial filter for a high-quality collimated beam. This collimated beam is cropped by a variable aperture A and then split into two perpendicular beams by the beam splitter BS1 with an intensity ratio of $50: 50$. The beam including the object $O$ in its optical path is referred to as the object beam and the other is the reference beam. Both the object beam and the reference beam are directed by mirrors M1 and M2, respectively, to the second beam splitter BS2. The angles at which they meet $\left(\theta_{x}\right.$ and $\left.\theta_{y}\right)$ at the camera sensor determine the fringe pattern of the hologram and the position of the twin image. The hologram is recorded using a customised real-time process analysis system developed at the Institute for Parallel and Distributed Systems. This system features a high-speed $1696 \times 1710$ pixel CMOS sensor with a pixel size of $8 \mu \mathrm{m}$. It allows for real-time imaging with an exposure time as low as $80 \mathrm{~ns}$ and frame rates up to $485 \mathrm{fps}$ at full resolution. The short exposure time makes the holographic set-up resistant to vibrations in the experimental set-up. Due to the huge volume of data generated by the sensor, it is connected to a powerful field-programmable gate array (FPGA) to process the data and to apply image processing algorithms, like 
holographic reconstructions, directly in the real-time process analysis system.

With the $x-y$ plane along the plane of the CMOS sensor and the $z$ axis perpendicular to the plane of the CMOS sensor, the intensity distribution in the plane of the hologram is given by $h(x, y, 0)=|O(x, y, 0)+R(x, y, 0)|^{2}$ (Picart and chang $\mathrm{Li}, 2013)$, where $O(x, y, 0)$ and $R(x, y, 0)$ represent the complex waves of the object beam and the reference beam in the hologram plane, respectively (for simplicity they are henceforth referred to as just $O$ and $R$ ). The intensity distribution can be expanded as $h=(O+R) \times(O+R)^{*}=$ $|O|^{2}+|R|^{2}+O R^{*}+R O^{*}$. In order to reconstruct the hologram, it is illuminated by the same reference wave as the one it was captured with. The reconstructed intensity of the image is given by

$$
\begin{aligned}
I & =h R=\left(|O|^{2}+|R|^{2}\right) R+O R^{*} R+R O^{*} R \\
& =\left(|O|^{2}+|R|^{2}\right) R+O|R|^{2}+O^{*} R^{2} .
\end{aligned}
$$

The reconstructed image has three components, as shown in Eq. (1). The first term represents the undiffracted wave passing through the hologram. It is the zero-order term, which only influences the overall brightness of the hologram. The second term represents the reconstructed object wave multiplied by a constant factor which forms the virtual image. The third term represents a defocussed real image of the object commonly referred to as the twin image. Among these three terms, only the second term contains useful information. To remove the zero-order component, a $3 \times 3$ pixel averaging filter is applied to the hologram and the result is subtracted from the original hologram. While reconstructing the resulting hologram, the zero-order component is removed but the twin image remains. The position of the twin image depends on the angles at which the object beam and the reference beam meet at the plane of the hologram $\left(\theta_{x}\right.$ and $\left.\theta_{y}\right)$. In the case of in-line holography (Gabor, 1948), $\theta_{x}$ and $\theta_{y}$ are set to $0^{\circ}$ and the twin image overlaps with the virtual image of the object. In the case of off-axis holography (Leith et al., 1965), the two angles $\left(\theta_{x}\right.$ and $\left.\theta_{y}\right)$ allow for the spatial separation of the twin image from the virtual image in the reconstructed plane. However, careful attention must be paid to the adjustment of $\theta_{x}$ and $\theta_{y}$, which must not exceed a maximum value $\theta_{\max }$ for which the carrier frequency of the hologram is equal to the Nyquist frequency of the sensor (Cuche et al., 2000):

$\theta_{x}, \theta_{y} \leq \arcsin \left(\frac{\lambda}{2 \Delta_{\mathrm{pix}}}\right)$

where $\lambda$ is the wavelength and $\Delta_{\text {pix }}$ is the pixel size of the CMOS sensor. In the presented set-up, $\theta_{\max }$ is determined to be $2.26^{\circ}$. The twin image is thus separated spatially by adjusting M1 and M2 along with the rotation of BS2 as shown Fig. 1 (top left).

\subsubsection{Digital reconstruction}

In digital holography, the captured hologram is reconstructed numerically at different planes in the direction of the object beam ( $z$ direction). The most commonly used reconstruction algorithms are the Fresnel transform, the angular spectrum and the convolution algorithm (Huaying et al., 2009). All of these algorithms are based on the fast Fourier transform (FFT) and are highly resource consuming when implemented on FPGAs. The Fresnel transform approach involves the computation of only one FFT, while the angular spectrum and convolution algorithm require the computation of two and three FFTs, respectively. As we intend to apply the system in real time on an FPGA with limited resources, we chose the Fresnel transform as the digital reconstruction algorithm. The reconstructed image $U(x, y, z)$ at a distance of $z$ obtained by the Fresnel transform is given by the equation

$$
\begin{aligned}
& U(x, y, z)=\frac{e^{i k z}}{i k z} \exp \left(\frac{i k}{2 z}\left(x^{2}+y^{2}\right)\right) \\
& \mathbf{F}\left\{h\left(x_{0}, y_{0}\right) \exp \left(\frac{i k}{2 z}\left(x_{0}^{2}+y_{0}^{2}\right)\right)\right\},
\end{aligned}
$$

where $h\left(x_{0}, y_{0}\right)$ is the hologram, $\lambda$ is the wavelength, $k$ is the wavenumber and $\mathbf{F}\{\}$ denotes the Fourier transform which is implemented digitally with the FFT. The computation involves three steps:

- multiplying the intensities of the hologram $h\left(x_{0}, y_{0}\right)$ with a complex exponential factor;

- computing a 2-D FFT of the product; and

- multiplying it with another complex exponential factor.

The size of the pixels in the reconstructed image at a distance $z$ is given in the literature by

$\Delta_{x}=\Delta_{y}=\frac{\lambda z}{\Delta_{\text {pix }} N}$,

where $\Delta_{\text {pix }}$ is the pixel size of the camera and $N$ is the number of pixels in a row or column. The reconstructed pixel size is directly proportional to $z$. Thus, reducing the distance of the object with respect to the camera results in a higher resolution. However, as $z$ decreases, the field of view also decreases and the spatial separation of the twin image becomes more difficult. Hence, a trade-off has to be achieved between the resolution, the measured area and the spatial separation of the twin image.

The reconstruction distance $z$ is given as an input parameter to the reconstruction algorithm. In order to find the position of the object along the $z$ axis, reconstruction is done over a range of values for $z$ and the distance at which the sharpest image is obtained is considered the depth of the object, i.e. the position of the object in the $z$ direction. In order to determine the sharpest image, a digital autofocussing algorithm is 

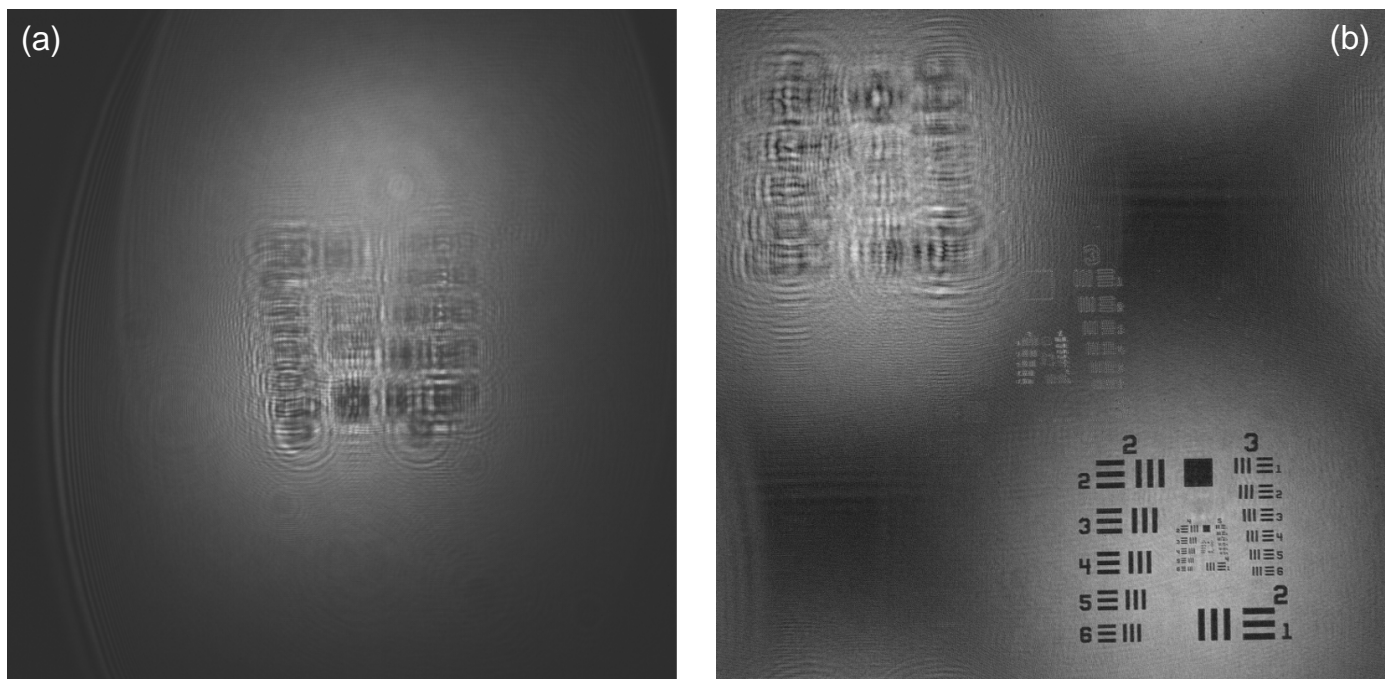

Figure 2. (a) A captured hologram of the USAF target and (b) a reconstruction of the hologram.

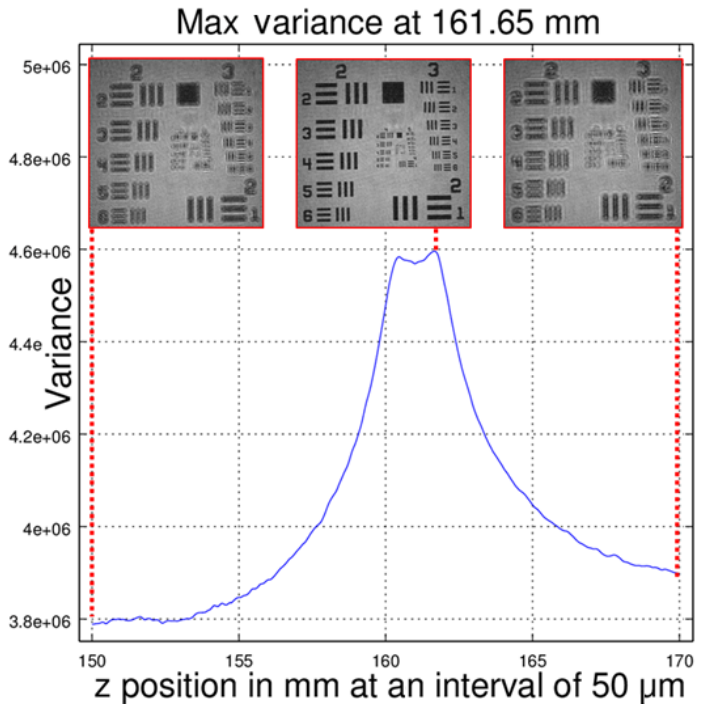

Figure 3. The variance plot of the hologram reconstruction in Fig. 2 at different planes in the $z$ direction.

used. Of the various autofocus evaluation functions, like the variance of grey level function, the weighted Fourier spectral function and the standard deviation correlation function, the variance of grey level is the most sensitive to detect sharp images (Wang et al., 2009). It is hence chosen to determine the depth of the object. With this method, the measure for the sharpness of an $N \times N$ image $I$ is given by the variance Var:

$\operatorname{Var}=\frac{1}{N^{2}} \sum_{m=1}^{N} \sum_{n=1}^{N}\left[I(m, n)-\frac{1}{N^{2}} \sum_{m=1}^{N} \sum_{n=1}^{N} I(m, n)\right]^{2}$.

The distance $z$ at which the reconstructed image has the maximum value of Var is the depth of the object.
In order to experimentally determine the resolution of the holographic system, a USAF target with elements from group 2 to group 7 was used as the object under testing. This USAF target was mounted on a three-axis linear stage with precision steps of $10 \mu \mathrm{m}$. The position of the mirrors M1 and M2 along with the rotation of the beam splitter BS2 were adjusted to obtain an optimum spatial separation of the twin image as explained in Sect. 2.1.

The hologram of the USAF target was captured by the CMOS sensor with an exposure time of $200 \mathrm{~ns}$ and is depicted in Fig. 2 (left) with a laser output power of $10 \mathrm{~mW}$. The combination of the laser output power and the exposure time of the CMOS sensor was chosen in such a way that a hologram with good contrast was recorded. Before reconstruction, the hologram was pre-processed to remove the zero-order term as explained in Sect. 2.1. The Fresnel transform was applied to obtain the reconstructed image presented in Fig. 2 (right). As discussed before, it contains the sharp virtual image and an unfocussed real twin image. However, due to the off-axis arrangement, they are spatially separated in the reconstructed plane.

For an automatic detection of depth, the autofocus variance measure was calculated only in the region of the virtual image and plotted for reconstructed images with different $z$ planes. This variance plot is shown in Fig. 3. The $z$ position, where the value of variance Var is maximum, reveals the depth of the object. In the above case, the maximum is at a distance of $161.65 \mathrm{~mm}$ in the $z$ direction with respect to the CMOS sensor. The two subpeaks in Fig. 3 correspond to the two surfaces of the USAF target, which has a substrate thickness of $1.5 \mathrm{~mm}$, and the line pair patterns $(120 \mu \mathrm{m}$ thick) are on the back surface of the substrate. Hence, the second peak corresponds to the USAF pattern and has a higher variance. 


\subsubsection{Accuracy and uncertainty in the position measurement}

A direct analysis of the resolution from the reconstructed image of the USAF target in Fig. 2 (right) reveals that the smallest line pairs which are distinguishable in both the horizontal and vertical direction are of group 5 element 3 . According to the USAF resolution charts, this corresponds to a system resolution of $40.3 \mathrm{lp} \mathrm{mm}^{-1}$. For the uncertainty in the position measurement of the bubble in the $x$ and $y$ direction, the size of the pixels in the reconstructed plane has to be derived experimentally $\left(\Delta_{x_{e}} \& \Delta_{x_{e}}\right.$ ) according to the Guide to the Expression of Uncertainty in Measurement (GUM; ISO, 2004 ) and shall be compared to the value $\left(\Delta_{\text {pix }}\right)$ in Eq. (4).

The accuracy of the position measurement depends on the pixel size in the reconstructed plane. The pixel size in both directions $\left(\Delta_{x_{e}}\right.$ and $\left.\Delta_{y_{e}}\right)$ in the reconstructed plane is calculated experimentally by counting the number of pixels corresponding to a fixed known length $D$. The uncertainty in the fixed known length $u(D)$ should also be known. By counting the number of pixels representing this fixed length $D$ in the reconstructed plane, the pixel size is determined. By repeating this process $i$ times, the average pixel count $\bar{p}$ and the uncertainty in the average pixel count $u(\bar{p})$ is calculated according to GUM type A with the following equation:

$u(\bar{p})=\frac{s(p)}{\sqrt{i}}$,

where $s(p)$ is the standard deviation of the values of $p$, and $i$ is the number of measurements. The experimental pixel size $\left(\Delta_{x_{e}}\right.$ and $\left.\Delta_{y_{e}}\right)$ in the reconstructed plane is given by

$\Delta_{x_{e}}=\Delta_{y_{e}}=f(D, p)=\frac{D}{\bar{p}}$.

According to the propagation of uncertainty in GUM (ISO, 2004), the uncertainty of $\Delta_{x_{e}}$ and $\Delta_{y_{e}}$, represented by $u\left(\Delta_{x_{e}}\right)$ and $u\left(\Delta_{y_{e}}\right)$, is given by

$$
\begin{aligned}
u\left(\Delta_{x_{e}}\right) & =u\left(\Delta_{y_{e}}\right) \\
& =\sqrt{\left(\frac{\partial f}{\partial D}\right)^{2} \times u^{2}(D)+\left(\frac{\partial f}{\partial \bar{p}}\right)^{2} \times u^{2}(\bar{p})} \\
& =\sqrt{\frac{u^{2}(D)}{\bar{p}} \times-\frac{D u^{2}(\bar{p})}{\bar{p}^{2}}}
\end{aligned}
$$

To experimentally determine the accuracy in the lateral plane, a custom target is used in place of the Taylor set-up. The target contains concentric circles of known sizes and has a structure tolerance of $0.5 \mu \mathrm{m}$ per edge. A hologram is captured with this target and the reconstructed image is analysed to determine $\Delta_{\text {pix }}$. One of these holograms and its reconstruction at the plane $z=155.850 \mathrm{~mm}$ is shown in Fig. 4 .

The fixed known length $D$ is taken to be the diameter of the largest circle in the reconstructed image in Fig. 4 (right).
Table 1. The number of pixels along the diameter.

\begin{tabular}{lcccc}
\hline Angles & $0^{\circ}$ & $45^{\circ}$ & $90^{\circ}$ & $135^{\circ}$ \\
\hline$p$ & 133 & 134 & 134 & 135 \\
\hline
\end{tabular}

The diameter of the largest circle is known to have a length $D$ of $1000 \mu \mathrm{m}$ and an uncertainty $u(D)$ of $1 \mu \mathrm{m}$. In order to count the number of pixels, $p$, corresponding to the diameter of this circle, the intensity profile of each row of the region containing the circle is plotted. A threshold value is determined using the Otsu (1979) method, and the pixels between the left and right edge on the circumference of the circle are counted. The row having the maximum number of pixels between the left and right edge on the circumference of the circle corresponds to the diameter of the circle. An intensity profile of the diameter row is plotted in Fig. 5.

The number of pixels along the horizontal axis $\left(0^{\circ}\right)$ was determined to be 134 . The diameter is also measured along three other axes at 45,90 and $135^{\circ}$. For this, the reconstructed image is rotated with the respective angle and the number of pixels along the diameter is obtained as explained previously. The angles were chosen in a way that minimises the interpolation errors caused by image rotation. The number of pixels along the diameter at the four different angles is shown in Table 1.

From Table 1, the average pixel count along the diameter $\bar{p}$ is calculated to be 134 pixels with a standard deviation $s(p)$ of 0.8165 pixels; $i$ is 4 . Therefore, from Eq. (6), $u(\bar{p})=0.4083 \mu \mathrm{m}$. The experimental pixel size $\left(\Delta_{x_{e}}\right.$ and $\left.\Delta_{y_{e}}\right)$ in the reconstruction plane is calculated from Eq. 7 to be $\Delta_{x_{e}}=\Delta_{y_{e}}=7.46 \mu \mathrm{m}$, and from Eq. (8) we calculate the uncertainty of $\Delta_{x_{e}}$ and $\Delta_{y_{e}}$ to be $u\left(\Delta_{x_{e}}\right)=u\left(\Delta_{y_{e}}\right)=0.02 \mu \mathrm{m}$. Hence, the experimentally calculated reconstructed pixel size is $\Delta_{x_{e}}=\Delta_{y_{e}}=7.46 \pm 0.02 \mu \mathrm{m}$, whereas the reconstructed pixel size $\left(\Delta_{x} \& \Delta_{y}\right)$ calculated from Eq. (4) is $\Delta_{x}=\Delta_{y}=$ $f(D, p)=7.58 \mu \mathrm{m}$, where $\lambda$ is $658 \mathrm{~nm}, z$ is $156.5 \mathrm{~mm}, \Delta_{\text {pix }}$ is $8 \mu \mathrm{m}$ and $N$ is 1696. It is observed that these values are in good agreement with the experimental values. The minor variation might result from instabilities in the laser wavelength and intensities.

For the accuracy of the position measurements in the $z$ direction, the object is kept fixed and repeated holograms were captured $j$ times. These holograms were reconstructed to determine the $z$ position of the object. Theoretically, they should have the same $z$ value. However, the experimentally determined depth deviates due to various factors like the laser wavelength fluctuation, laser intensity fluctuation, vibration and image noise. From the $j$ number of extracted $z$ values, the uncertainty in the position measurements along the $z$ direction $u(z)$ is given by the standard deviation $s(z)$ :

$$
u(z)=s(z)=\sqrt{\frac{\sum_{k=1}^{j}\left(z_{k}-\bar{z}\right)^{2}}{j-1}},
$$



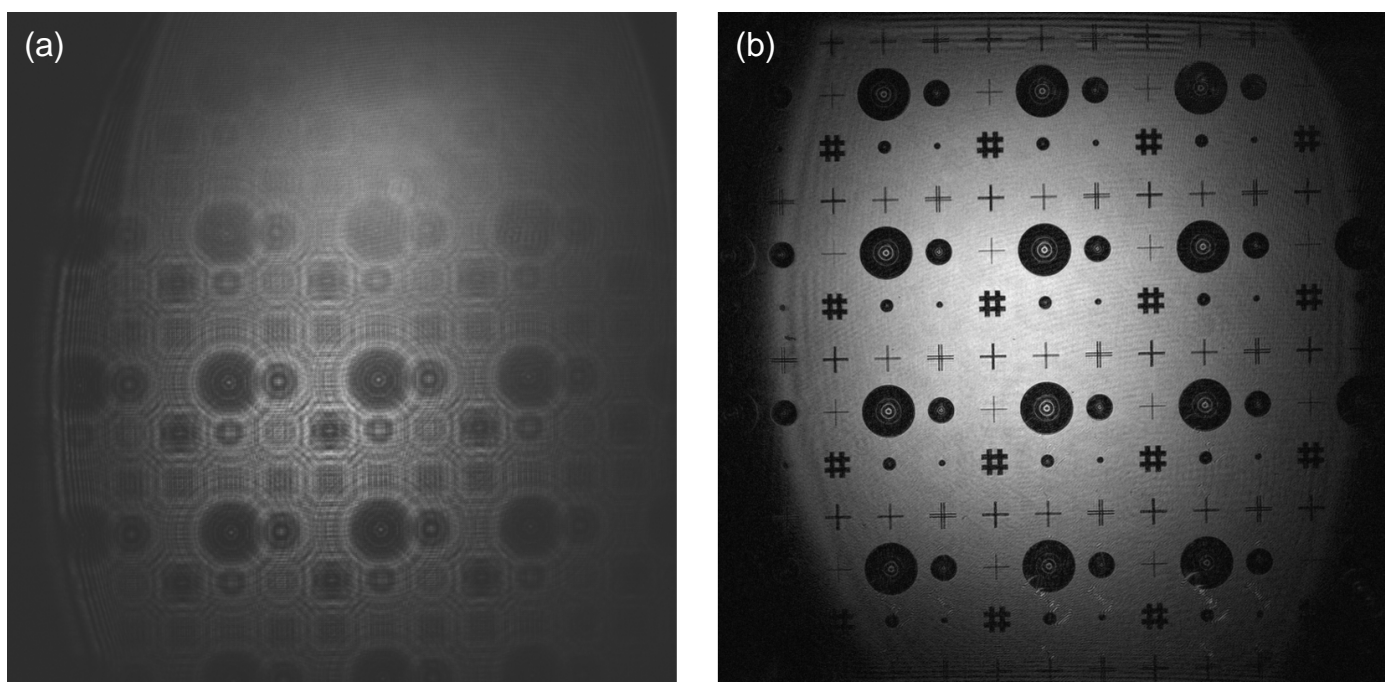

Figure 4. (a) A captured hologram of the custom target and (b) a reconstruction of the hologram.

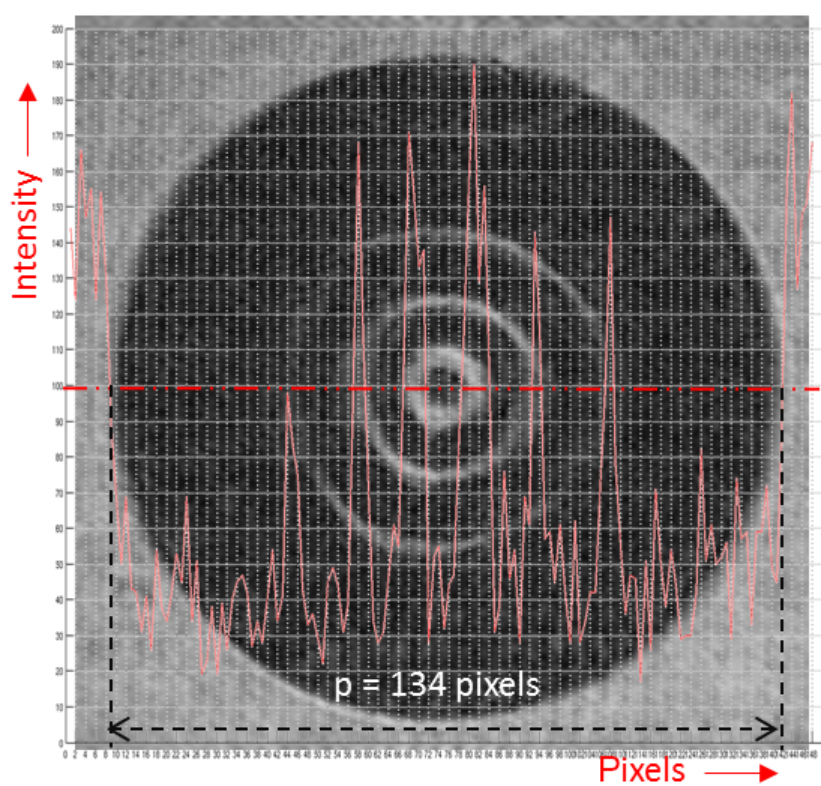

Figure 5. The intensity profile of the row corresponding to the diameter of the circle.

where $\bar{z}$ is the mean of the extracted $z$ values.

Repeatedly, 100 holograms were captured with the target kept fixed $(j=100)$. For each hologram, reconstruction and autofocussing was performed to determine the position of the object along the $z$ axis. The raw experimental data are presented in the Appendix in Table A1. The mean $z$ position was calculated to be $153.15 \mathrm{~mm}$ with a standard deviation, $s(z)$, of $0.012 \mathrm{~mm}$. From Eq. (9), the uncertainty in the position measurement along the $z$ direction $u(z)$ is calculated to be $u(z)=11.94 \mu \mathrm{m}$.

\subsection{Laser Raman-spectroscopy subsystem}

The confocal Raman-spectroscopy subsystem uses a pulsed $\mathrm{Nd}$ :YAG laser with a wavelength of $532 \mathrm{~nm}$. The pulsed laser is operated at $5 \mathrm{~Hz}$ of frequency to provide thermal stability. The set-up of the subsystem is illustrated in Fig. 1 (bottom left). The pulses are directed through a microscope via an open light path with dielectric and dichroic mirrors (DM in Fig. 1; bottom left). An objective, L3 in Fig. 1 (bottom left), with a magnification factor of 40 is used to focus the laser light into a fixed spot ( $\mathrm{F}$ in Fig. 1; bottom left) in the microchannel. The $40 \times$ objective possesses a working distance of $4 \mathrm{~mm}$; see Fig. 6 (left). It is also possible to replace the latter with a $4 \times$ objective, shown in Fig. 6 (right), in order to facilitate focussing on the right spot. The maximum optical energy focussed at this spot was experimentally determined to be approximately $5 \mathrm{~mJ}$. This focal spot, $\mathrm{F}$, is within the area visible in the reconstructed holograms from the Mach-Zehnder holography subsystem. The position of $\mathrm{F}$ in the reconstructed hologram is determined by calibration. Since a $360^{\circ}$ arrangement is used, the scattered light, Rayleigh scatter and wavelength-shifted Raman scatter, are directed back on the same optical path. The Rayleigh scatter is separated from the Raman scatter with a dichroic mirror (DM), which reflects the light with a wavelength of $532 \mathrm{~nm}$. After the dichroic mirror, the Raman light is coupled into an optical fibre (OF) with a core diameter of $50 \mu \mathrm{m}$. The optical fibre leads the Raman light into the spectrometer. The diameter of the fibre acts as a pinhole to obtain a confocal arrangement for a good depth resolution. The spatial resolution was checked before experimentation using a continuouswave laser, instead of the pulsed laser, with a similar arrangement. It was determined to be about $5 \mu \mathrm{m} \times 5 \mu \mathrm{m}$ in the lateral direction and $40 \mu \mathrm{m}$ in depth (Fräulin et al., 2014). A thermoelectrically cooled CCD camera is connected to the 

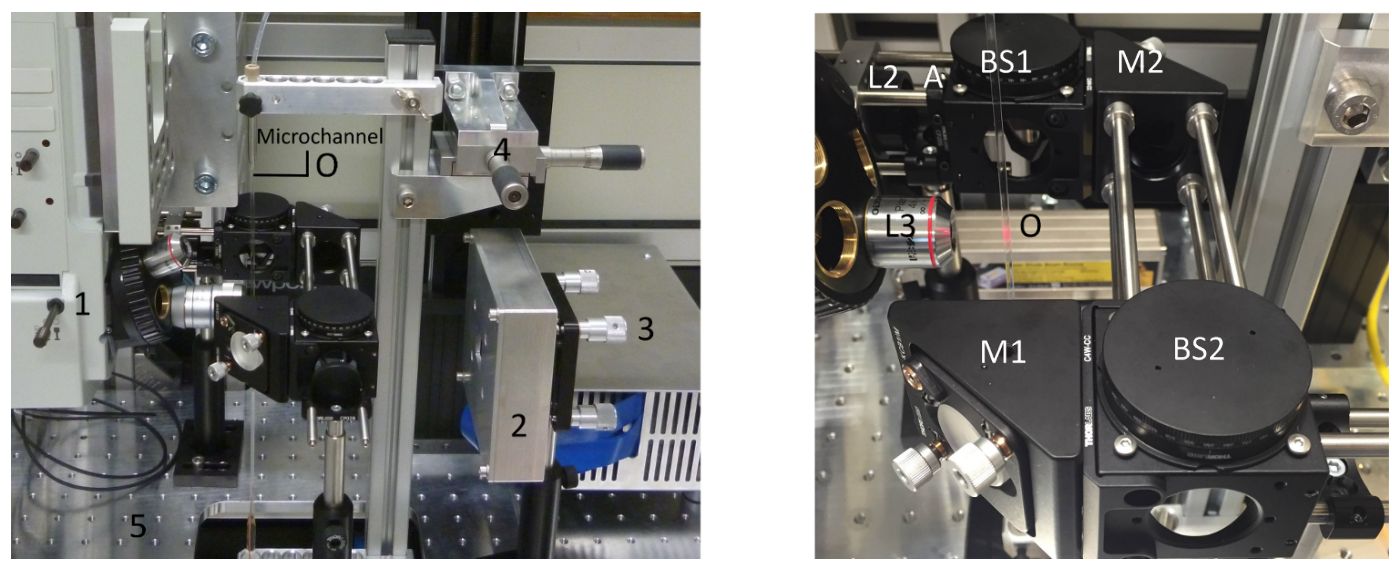

Figure 6. The experimental set-up for the combined Mach-Zehnder holography and confocal Raman-spectroscopy sensor system to investigate the bubble dynamics and the localised concentration measurement simultaneously in a Taylor flow.

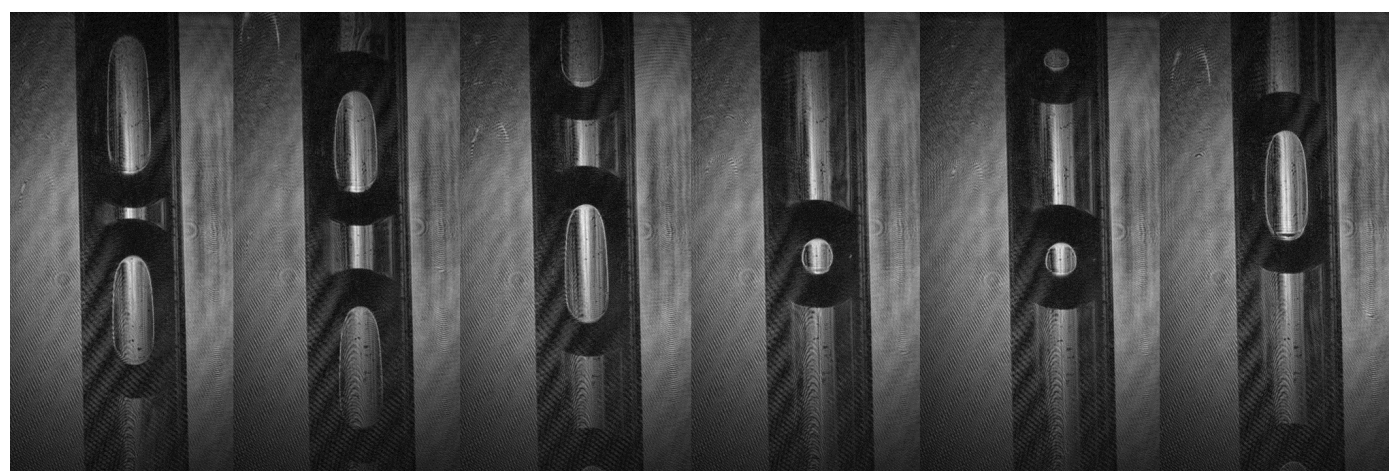

Figure 7. The reconstructed holograms from the experimental set-up shown in Fig. 6 from which the bubble position is extracted.

spectrometer. The data are transferred to the "WinSpec" software (Princeton Instruments, a division of Roper Scientific, Inc., Trenton, NJ 08619), which allows for the triggering of the spectrometer to single laser pulses. Another CCD camera is mounted on the microscope tube to capture an image of the place where the focus is located. This is used for calibration purposes to determine the exact position of the focal spot in the reconstructed hologram.

The sensitivity of the Raman system for small signals is determined by the limit of detection, LOD, given by

$\mathrm{LOD}=\frac{N_{3 \delta}}{I(i)} c(i)$,

where $I(i)$ is the intensity of the characteristic signal of component $i, c(i)$ is its concentration and $N_{3 \delta}$ is the noise calculated by multiplying the standard deviation $\delta$ of the spectra in a region without any characteristic signal by a factor of 3 .

\section{Experimental results}

Both the digital Mach-Zehnder holography subsystem and the confocal Raman-spectroscopy subsystem were integrated and constructed into a single synchronised system as shown in Fig. 6. The left image in Fig. 6 shows the overall system, while the right image in Fig. 6 shows a zoomed-in view. In Fig. 6 (left), 1 depicts the microscope for confocal Raman spectroscopy, 2 depicts the CMOS sensor and 3 depicts the FPGA. The microchannel of the Taylor flow setup, marked as $\mathrm{O}$ in Fig. 6, was mounted on a movable stage, which is 4 in Fig. 6. For the direction in the plane of the microchannel cross section, micrometre adjusting screws are used. The height is tuned on a threaded rod. The entire set-up was mounted on a vibration-stabilised optical bench (5). The naming conventions of the components in Fig. 6 (right) are in correspondence with Fig. 1.

First, the bubble dynamics measurements were conducted with air and water in the microchannel (Sect. 3.1). Thereafter, experiments were performed to analyse the $\mathrm{CO}_{2}$ absorption in caustic soda to verify the functionality of the entire system as a whole (Sect. 3.2). The results are presented and discussed in the subsections below. 

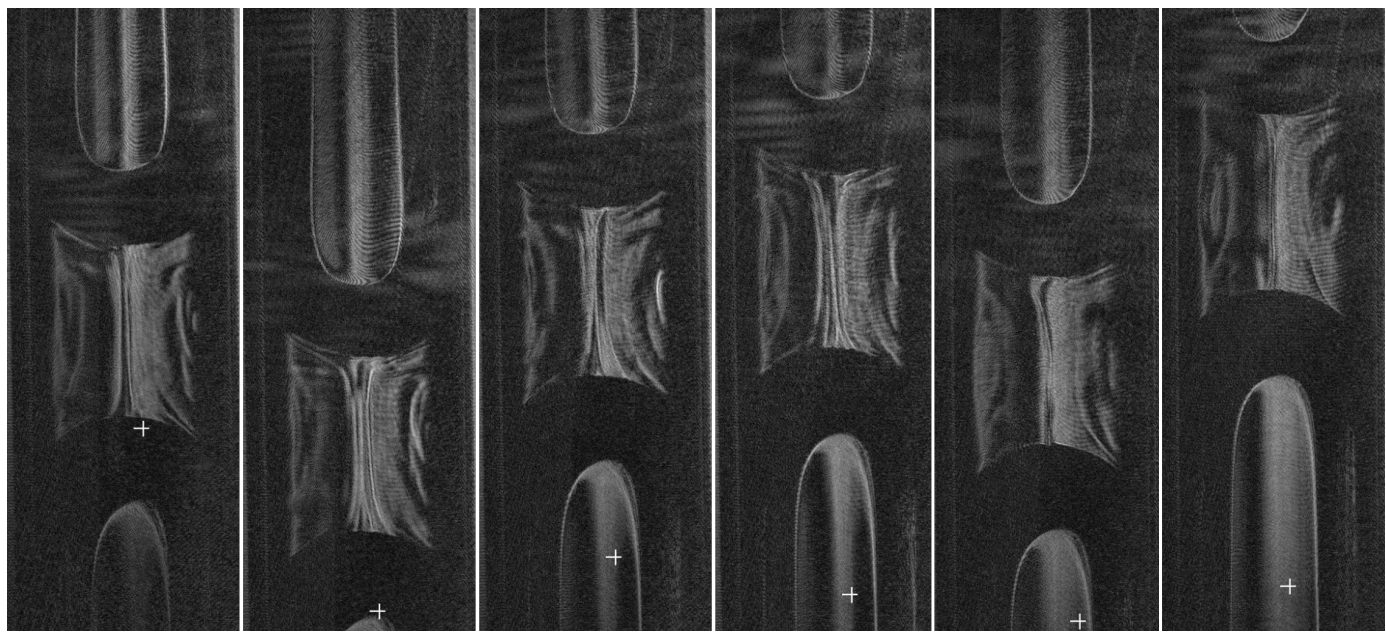

Figure 8. The reconstructed holograms from the experiments with $\mathrm{CO}_{2}$ absorption in caustic soda showing the mixing and turbulence of the chemical compounds.

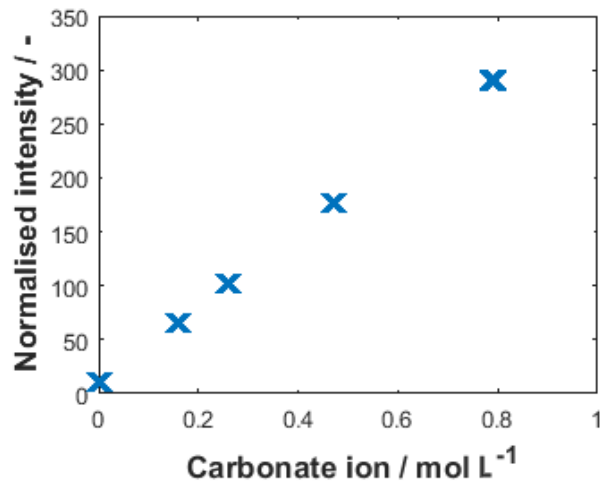

Figure 9. The calibration plot for the known $\mathrm{CO}_{3}^{-2}$ concentration against the normalised intensity.

\subsection{Bubble dynamics}

Water and air were used as the liquid and the gaseous phase, respectively, and bubbles with sizes from $1-5 \mathrm{~mm}$ were induced into the microchannel. This Taylor flow set-up was placed within the object beam of the holographic set-up, and holograms were captured.

The reconstruction of the hologram was done at a range of $z$ planes within the microchannel, and the autofocussing algorithm was used to select the plane containing the rearmost point of the tailing edge of the bubble lying on the interface between the gaseous and liquid phase; this is hereafter referred to as the lowermost point of the bubble. Such reconstructed planes of the bubbles in the Taylor flow are shown in Fig. 7. The depth of these planes corresponds to the $z$ position of the lowermost point of the bubble. For the $x$ and $y$ positions of the lowermost point of the bubble, edge detection with image processing algorithms was performed to find the pixel corresponding to the lowermost point of the bubble; the actual position is given by multiplying this pixel position with the pixel pitch of the reconstructed plane (as given by Eq. 4). Hence, the 3-D position of the lowermost point of the bubble in the microchannel is determined.

Apart from the ability to measure the 3-D position, the digital Mach-Zehnder holography technique has another major advantage. It is a lensless measurement technique, and hence the need for expensive lenses and the practical focussing problems of shadowgraphy are completely eliminated. Although this technique involves a computation-intensive reconstruction step, it does not limit the speed of tracking the bubble position as the holograms are recorded continuously and the reconstruction is a post-process independent of capturing the holograms.

Furthermore, it was observed that during the investigation of $\mathrm{CO}_{2}$ absorption in caustic soda, the mixing and turbulence in the fluid phase of the reaction are visible in the reconstructed hologram as seen in Fig. 8. They result from the inner circulations within the slug in the wake of the bubble (Gupta et al., 2010; Sobieszuk et al., 2012; Talimi et al., 2012), thus creating a concentration profile of the carbonate ion within the slug. This is visible due to the change in the refractive index, but it cannot be quantitatively analysed with high spatial resolution, since it is an integrated value along the depth of the capillary. Thus, this technique may also be used to qualitatively study the behaviour of mixing and turbulence in bubbly flows. It was often observed that the lowermost point of the bubble was not exactly in the centre of the microchannel, and the turbulence was asymmetrical; hence the need for 3-D measurements.

\subsection{Simultaneous concentration measurements}

Concentration measurements with the confocal Raman subsystem were performed with $\mathrm{CO}_{2}$ absorption in caustic soda. 

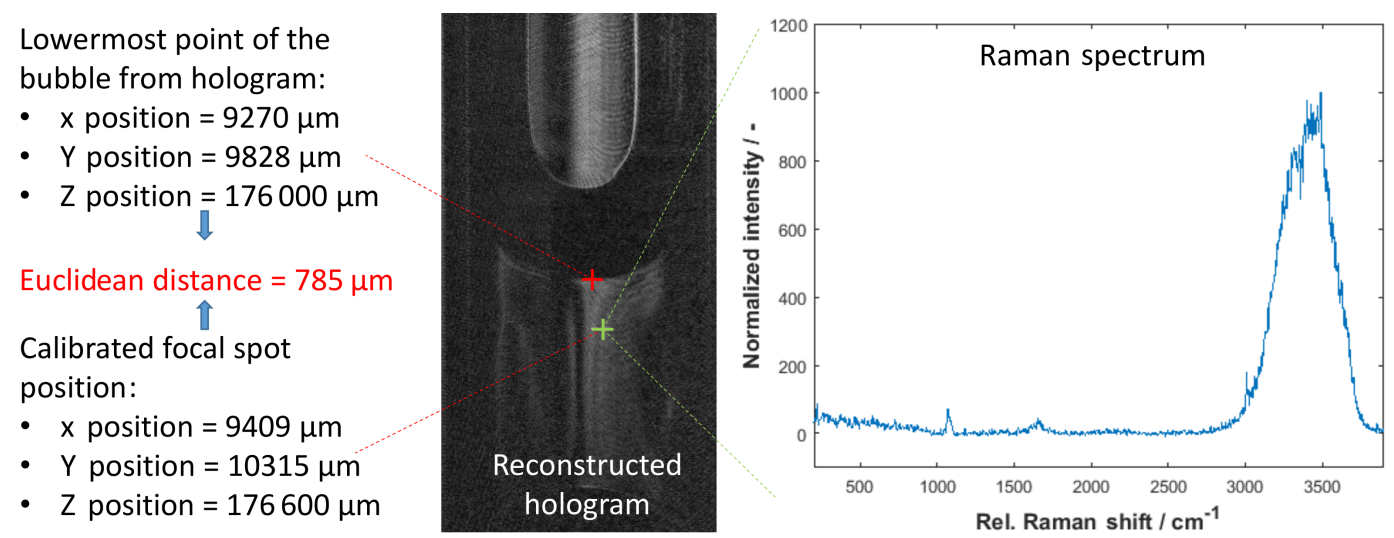

Figure 10. The experimental results of the simultaneous bubble position measurement and localised concentration measurement of $\mathrm{CO}_{2}$ absorption in caustic soda.

As the bubble rises along the microchannel, $\mathrm{CO}_{2}$ is absorbed. The bubble length hence decreases. Under the assumption that the $\mathrm{pH}$ is higher than 12 , the absorbed $\mathrm{CO}_{2}$ reacts rapidly to the carbonate ion $\mathrm{CO}_{3}^{-2}$ according to the carbonate equilibrium. The Raman spectrum shown in Fig. 10 is taken with one single laser pulse. The energy of one single laser pulse was experimentally measured to be $5.1 \mathrm{~mJ}$ after the objective in front of the microchannel. For a first test, we positioned the microchannel using the adjustment screws in such a way that the laser focus is positioned in the middle of the cross section at a distance of $207 \mathrm{~mm}$ from the gas-liquid contacting.

The simultaneous measurement of the 3-D bubble position and the localised concentration measurement is presented in Fig. 10. The spectrum on the right side of Fig. 10 is shaped by a broad characteristic signal of the aqueous solution at 3200 to $3800 \mathrm{~cm}^{-1}$. This signal was used to normalise the spectra.

The characteristic signal of the carbonate ion is detected at a wave number of $1080 \mathrm{~cm}^{-1}$. It was calibrated by taking the Raman spectra of samples with a known concentration of the carbonate ion. According to Fig. 9, this resulted in a linear dependency of the concentration on the intensity. Thus, the concentration in the spectrum shown in Fig. 10 is determined to be $0.19 \mathrm{molL}^{-1}$. The position of the lowermost point of the 3-D bubble was extracted from the hologram as 9270 , 9828 and $176000 \mu \mathrm{m}$, and the 3-D position of the focal spot for the concentration measurement was determined by calibration to be 9409,10315 and $176600 \mu \mathrm{m}$. From this, the Euclidean distance between the lowermost point of the bubble and the focal spot, where the concentration measurement was performed, is calculated to be $785 \mu \mathrm{m}$. Thus, the concentration of the carbonate ion at the focal spot is $0.19 \mathrm{molL}^{-1}$ with the correlated 3-D distance between the focal spot and the lowermost point of the bubble being $785 \mu \mathrm{m}$.

Several spectra were taken at the same position. The mean concentration of the carbonate ion was determined to be about $0.20 \mathrm{molL}^{-1} \pm 0.03 \mathrm{molL}^{-1}$. The sensitivity of the Raman system for small signals is determined by the LOD according to Eq. (10). The standard deviation $\delta$ for one single spectrum is about $5.6 \mathrm{cts}$. With a mean intensity of the carbonate signal of $69.5 \mathrm{cts}$, this results in an LOD of $0.05 \mathrm{molL}^{-1}$.

\section{Conclusions}

A noninvasive sensor system for the simultaneous in situ characterisation of bubble dynamics and spatially resolved concentration measurements is presented in this paper. The sensor system is a combination of two subsystems tuned to investigate bubbly flows. The digital Mach-Zehnder holography subsystem performs the measurement of real-time bubble dynamics, while the confocal Raman-spectroscopy subsystem measures the concentration at a localised spot.

Although measuring the dynamics of the bubble with a digital Mach-Zehnder holographic technique requires a computation-intensive reconstruction step, it provides a unique capability to extract the 3-D position of the bubble with a single camera set-up. As a lensless technique, it is not limited to bubbles in one single plane. Hence, it allows for the detection of freely moving bubbles within the observed 3-D volume. This is a further advantage as the bubbles generally have random motions. The high-speed CMOS sensor attached to an FPGA in the system achieves a very low exposure time $(200 \mathrm{~ns})$, making the system fast, robust and immune to vibrations which otherwise might be a highly degrading factor in holography. The experimental results showed that the reconstructed image resolution for the 3 -D position measurement was lower than $8 \mu \mathrm{m}$ in the $x$ and $y$ direction. The uncertainty in the $z$ position measurement of the system was found to be lower than $\pm 12 \mu \mathrm{m}$. It was also shown that mixing or turbulence in the liquid phase may also be studied successfully with the holographic subsystem.

The confocal Raman-spectroscopy subsystem utilises a pulsed Nd:YAG laser to measure the localised concentration of chemical products around the bubble. This subsys- 
tem offers a highly selective, spatially resolved and noninvasive technique for concentration measurements. The limit of detection of this subsystem was experimentally found to be $0.05 \mathrm{molL}^{-1}$.

Both subsystems were integrated and built into a singlespace constrained system, and they were synchronised with each other. This system was applied to analyse the absorption of $\mathrm{CO}_{2}$ in caustic soda in the Taylor flow. The experimental results of simultaneous measurements of the concentration at a localised spot and the correlated distance of this spot to the bubble, in 3-D, are presented in this paper. This sensor system opens a myriad of possibilities to measure concentration profiles around bubbles by acquiring a set of data points with the concentration measurement and the correlated distance to the bubble.

Data availability. The data have been presented in Appendix A. 
Appendix A: Experimental data for accuracy measurement along the $z$ direction

Table A1 presents the experimental data for the accuracy measurement along the $z$ direction. Repeatedly, 100 holograms $(j=100)$ were captured with the target fixed. The $z$ positions were then extracted after reconstructing these holograms and applying an autofocus algorithm onto them. Theoretically, they should all have the same $z$ position. However, the experimentally determined depth deviates due to various factors like the laser wavelength fluctuation, laser intensity fluctuation, vibration and image noise.

Table A1. Reconstructed object positions along the $z$ axis of 100 holograms without moving the target.

\begin{tabular}{lrrrrrrr}
\hline Repetition $j$ & $z$ position & Repetition $j$ & $z$ position & Repetition $j$ & $z$ position 1 & Repetition $j$ & $z$ position \\
\hline 1 & 153.156 & 26 & 153.151 & 51 & 153.142 & 76 & 153.147 \\
2 & 153.146 & 27 & 153.147 & 52 & 153.153 & 77 & 153.137 \\
3 & 153.142 & 28 & 153.136 & 53 & 153.148 & 78 & 153.134 \\
4 & 153.151 & 29 & 153.151 & 54 & 153.139 & 79 & 153.136 \\
5 & 153.163 & 30 & 153.160 & 55 & 153.135 & 80 & 153.137 \\
6 & 153.120 & 31 & 153.135 & 56 & 153.151 & 81 & 153.160 \\
7 & 153.132 & 32 & 153.148 & 57 & 153.149 & 82 & 153.144 \\
8 & 153.167 & 33 & 153.145 & 58 & 153.143 & 83 & 153.151 \\
9 & 153.136 & 34 & 153.152 & 59 & 153.140 & 84 & 153.137 \\
10 & 153.162 & 35 & 153.143 & 60 & 153.139 & 85 & 153.142 \\
11 & 153.142 & 36 & 153.121 & 61 & 153.124 & 86 & 153.142 \\
12 & 153.139 & 37 & 153.138 & 62 & 153.154 & 87 & 153.159 \\
13 & 153.176 & 38 & 153.126 & 63 & 153.169 & 88 & 153.148 \\
14 & 153.140 & 39 & 153.141 & 64 & 153.131 & 89 & 153.159 \\
15 & 153.168 & 40 & 153.137 & 65 & 153.159 & 90 & 153.143 \\
16 & 153.169 & 41 & 153.154 & 66 & 153.121 & 91 & 153.168 \\
17 & 153.141 & 42 & 153.134 & 67 & 153.129 & 92 & 153.167 \\
18 & 153.156 & 43 & 153.141 & 68 & 153.147 & 93 & 153.127 \\
19 & 153.131 & 44 & 153.166 & 69 & 153.177 & 94 & 153.139 \\
20 & 153.136 & 45 & 153.144 & 70 & 153.146 & 95 & 153.142 \\
21 & 153.149 & 46 & 153.138 & 71 & 153.153 & 96 & 153.149 \\
22 & 153.143 & 47 & 153.152 & 72 & 153.150 & 97 & 153.154 \\
23 & 153.128 & 48 & 153.154 & 73 & 153.133 & 98 & 153.140 \\
24 & 153.141 & 49 & 153.141 & 74 & 153.144 & 99 & 153.141 \\
25 & 153.149 & 50 & 153.148 & 75 & 153.156 & 100 & 153.147 \\
\hline
\end{tabular}

$Z$ positions in $\mathrm{mm}$. 
Competing interests. The authors declare that they have no conflict of interest.

Acknowledgements. We gratefully thank the German Research Foundation within the priority programme 1740, "Reactive Bubbly Flows", for supporting this work under grant numbers SI 587/11-1 and RI 2512/1-1. We would also like to thank Tobias Haist from the Institut für Technische Optik, Universität Stuttgart, for his valuable discussions.

Edited by: M. J. da Silva

Reviewed by: two anonymous referees

\section{References}

Angeli, P. and Gavriilidis, A.: Hydrodynamics of Taylor flow in small channels: a review, J. Mech. Eng. Sci., 222, 737-751, doi:10.1243/09544062JMES776, 2008.

Cuche, E., Marquet, P., and Depeursinge, C.: Spatial filtering for zero-order and twin-image elimination in digital off-axis holography, Appl. Opt., 39, 4070-4075, doi:10.1364/AO.39.004070, 2000.

Deckwer, W.-D.: Bubble column reactors, Wiley, Chichester, 1992.

Deen, N. G., Mudde, R. F., Kuipers, J. A. M., Zehner, P., and Kraume, M.: Bubble Columns, in: Ullmann's encyclopedia of industrial chemistry, Wiley-VCH Verlag GmbH \& Co. KGaA, Weinheim, 25, 359-379, doi:10.1002/14356007.b04_275.pub2, 2012.

Desse, J.-M. and Olchewsky, F.: Digital Mach-Zehnder Holographic Interferometer Using Pulsed Laser for Analyzing Large Flow Fields, in: Imaging and Applied Optics 2016, p. DW1H.6, Optical Society of America, doi:10.1364/DH.2016.DW1H.6, 2016.

Ferrara, M. A., Di Caprio, G., Managò, S., De Angelis, A., Sirleto, L., Coppola, G., and De Luca, A. C.: Label-Free Imaging and Biochemical Characterization of Bovine Sperm Cells, Biosensors, 5, 141-157, doi:10.3390/bios5020141, 2015.

Ferrara, M. A., Angelis, A. D., Luca, A. C. D., Coppola, G., Dale, B., and Coppola, G.: Simultaneous Holographic Microscopy and Raman Spectroscopy Monitoring of Human Spermatozoa Photodegradation, IEEE J. Sel. Top. Quant., 22, 27-34, doi:10.1109/JSTQE.2015.2496265, 2016.

Fletcher, P. D. I., Haswell, S. J., and Zhang, X.: Monitoring of chemical reactions within microreactors using an inverted Raman microscopic spectrometer, Electrophoresis, 24, 3239-3245, doi:10.1002/elps.200305532, 2003.

Fräulin, C., Rinke, G., and Dittmeyer, R.: In-situ Laser Raman Spectroscopy Adapted to Process Conditions for Studying Cyclohexane Oxidation, J. Flow Chem., 3, 87-91, doi:10.1556/JFCD-13-00007, 2013.

Fräulin, C., Rinke, G., and Dittmeyer, R.: Characterization of a new system for space-resolved simultaneous in situ measurements of hydrocarbons and dissolved oxygen in microchannels, Microfluid. Nanofluid., 16, 149-157, doi:10.1007/s10404-0131223-8, 2014.

Gabor, D.: A new microscopic principle, Nature, 161, 777-778, 1948.
Gebauer, F., Hlawitschka, M. W., and Bart, H.-J.: CFD aided investigation of single droplet coalescence, Chinese J. Chem. Eng., 24, 249, doi:10.1016/j.cjche.2015.07.024, 2016.

Gupta, R., Fletcher, D. F., and Haynes, B. S.: Taylor Flow in Microchannels: A Review of Experimental and Computational Work, The Journal of Computational Multiphase Flows, 2, 1-32, doi:10.1260/1757-482X.2.1.1, 2010.

Huaying, W., Baoqun, Z., and Wei, L.: Comparison of the reconstruction algorithms in digital micro-holography, in: Intelligent Computing and Intelligent Systems, ICIS 2009, IEEE International Conference on, 2, 838-842, doi:10.1109/ICICISYS.2009.5358270, 2009.

ISO: Guide to the expression of uncertainty in measurement (GUM)-Supplement 1: Numerical methods for the propagation of distributions, vol. ISO draft guide DGUIDE99998, International Organization for Standardization, Geneva, 2004.

Jakobsen, H. A.: Chemical Reactor Modeling - Multiphase Reactive Flows, Springer Science, 2008.

Kashid, M. N., Renken, A., and Kiwi-Minsker, L.: Gas-liquid and liquid-liquid mass transfer in microstructured reactors, Chem. Eng. Sci., 66, 3876-3897, doi:10.1016/j.ces.2011.05.015, 2011.

Kessler, R. W., Kessler, W., and Zikulnig-Rusch, E.: A Critical Summary of Spectroscopic Techniques and their Robustness in Industrial PAT Applications, Chemie Ingenieur Technik, 88, 710-721, doi:10.1002/cite.201500147, 2016.

Kim, M. K.: Principles and techniques of digital holographic microscopy, Journal of Photonics for Energy, 018005-018005-50, doi:10.1117/6.0000006, 2010.

Lebrun, D., Allano, D., Méès, L., Walle, F., Corbin, F., Boucheron, R., and Fréchou, D.: Size measurement of bubbles in a cavitation tunnel by digital in-line holography, Appl. Opt., 50, H1-H9, doi:10.1364/AO.50.0000H1, 2011.

Leith, E. N., Upatnieks, J., and Haines, K. A.: Microscopy by Wavefront Reconstruction*, J. Opt. Soc. Am., 55, 981-986, doi:10.1364/JOSA.55.000981, 1965.

Lewis, I. R. (Ed.): Handbook of Raman spectroscopy: from the research laboratory to the process line, Practical spectroscopy, 28, Marcel Dekker, New York, 2001.

Lindken, R. and Merzkirch, W.: A novel PIV technique for measurements in multiphase flows and its application to two-phase bubbly flows, Exp. Fluids, 33, 814-825, doi:10.1007/s00348002-0500-1, 2002.

Micó, V., Ferreira, C., Zalevsky, Z., and García, J.: Basic principles and applications of digital holographic microscopy, in: Microscopy - Science, Technology, Applications and Education, edited by: Méndez-Vilas, A. and Díaz, J., Formatex Research Center, 2, 1411-1418, 2010.

Orlov, S. S., Abarzhi, S. I., Oh, S. B., Barbastathis, G., and Sreenivasan, K. R.: High-performance holographic technologies for fluid-dynamics experiments, Philos. T. R. Soc. A, 368, 17051737, doi:10.1098/rsta.2009.0285, 2010.

Otsu, N.: A Threshold Selection Method from GrayLevel Histograms, IEEE T. Syst. Man Cyb., 9, 62-66, doi:10.1109/tsmc.1979.4310076, 1979.

Pavillon, N. and Smith, N. I.: Implementation of simultaneous quantitative phase with Raman imaging, EPJ Techniques and Instrumentation, 2, 1, doi:10.1140/epjti/s40485-015-0015-9, 2015.

Picart, P. and chang Li, J.: Digital Holography, Wiley-ISTE, 2013. 
Rantanen, J.: Process analytical applications of Raman spectroscopy, The J. Pharm. Pharmacol., 59, 171-7, doi:10.1211/jpp.59.2.0004, 2007.

Rinke, G., Ewinger, A., Kerschbaum, S., and Rinke, M.: In situ Raman spectroscopy to monitor the hydrolysis of acetal in microreactors, Microfluid. Nanofluid., 10, 145-153, doi:10.1007/s10404-010-0654-8, 2011.

Sathe, M. J., Thaker, I. H., Strand, T. E., and Joshi, J. B.: Advanced PIV/LIF and shadowgraphy system to visualize flow structure in two-phase bubbly flows, Chem. Eng. Sci., 65, 2431-2442, doi:10.1016/j.ces.2009.11.014, 2010.

Sobieszuk, P., Aubin, J., and Pohorecki, R.: Hydrodynamics and Mass Transfer in Gas-Liquid Flows in Microreactors, Chem. Eng. Technol., 35, 1346-1358, doi:10.1002/ceat.201100643, 2012.

Talimi, V., Muzychka, Y., and Kocabiyik, S.: A review on numerical studies of slug flow hydrodynamics and heat transfer in microtubes and microchannels, Int. J. Multiphas. Flow, 39, 88-104, doi:10.1016/j.ijmultiphaseflow.2011.10.005, 2012.

Tanaka, Y. and Murata, S.: Three-dimensional Visualization of Hydrogen Bubble Method by means of Digital Holography Using Two-wavelength Reconstruction, Journal of the Japanese Society for Experimental Mechanics, 12, s121-s125, doi:10.11395/jjsem.12.s121, 2012.
Thoroddsen, S. T., Etoh, T. G., and Takehara, K.: High-speed imaging of drops and bubbles, Annu. Rev. Fluid Mech., 40, 257-285, doi:10.1146/annurev.fluid.40.111406.102215, 2008.

Toker, G. R.: Holographic Interferometry: A Mach-Zehnder Approach, CRC Press, 2016.

Tokuhiro, A., Fujiwara, A., Hishida, K., and Maeda, M.: Measurement in the wake region of two bubbles in close proximity by combined shadow-image and PIV techniques, J. Fluid. Eng., 121, 191-197, 1999.

Vankeirsbilck, T., Vercauteren, A., Baeyens, W., and Weken, G. V. D.: Applications of Raman spectroscopy in pharmaceutical analysis Applications of Raman spectroscopy in pharmaceutical analysis, Trends Anal. Chem., 21, 869-877, doi:10.1016/S01659936(02)01208-6, 2015.

Versluis, M.: High-speed imaging in fluids, Exp. Fluids, 54, 1458, doi:10.1007/s00348-013-1458-x, 2013.

Wang, H., Qin, A., and Huang, M.: Autofocus Method for Digital Holographic Reconstruction of Microscopic Object, in: 2009 Symposium on Photonics and Optoelectronics, 1-4, doi:10.1109/SOPO.2009.5230170, 2009. 